\title{
Misdiagnoses of transthyretin amyloidosis: a clinical and electrodiagnostic study
}

\author{
Andrea Cortese ${ }^{1 *}$, Alessandro Lozza', Elisa Vegezzi ${ }^{1}$, Enrico Alfonsi ${ }^{1}$, Arrigo Moglia', Giampaolo Merlini ${ }^{2}$, \\ Laura Obici ${ }^{2}$ \\ From First European Congress on Hereditary ATTR amyloidosis \\ Paris, France. 2-3 November 2015
}

\section{Background}

Misdiagnosis of ATTR and late diagnosis may be detrimental hampering adequate management and delaying therapy onset. Objective of the present study was to investigate in a large single-centre cohort of geneticallyconfirmed ATTR patients the prevalence, type and causes of misdiagnoses. Given the high frequency of cases erroneously diagnosed as having chronic inflammatory demyelinating polyneuropathy (CIDP), we investigated the electrodiagnostic (EDx) features which can help distinguish ATTR from CIDP.

\section{Methods}

Retrospective study design. Review of clinical notes and EDx studies of ATTR patients referred to Amyloid Research and Treatment Centre and C. Mondino National Neurological Institute (Pavia) between 1999 and 2013. EDx of thirty-five patients diagnosed with CIDP were used as control for comparison.

\section{Results}

Out of 150 patients with ATTR 51(32\%) were initially misdiagnosed including 30(59\%) CIDP and 11(22\%) lumbar spinal stenosis. Eleven (22\%) patients underwent spine surgery and $38(74 \%)$ were treated with immunotherapies. Patients misdiagnosed had a significant longer delay before diagnosis of $47 \pm 3.7$ months vs $34 \pm 2.7$ months ( $\mathrm{p}=0.01$ ). Lack of family history and onset after 56 years were significantly associated with misdiagnosis $(\mathrm{p}<0.01)$. Out of 30 patients misdiagnosed as having CIDP, 17 had original EDx available for review. Six (35\%) had definite and 3 (17\%) possible CIDP according to EFNS criteria, while 8 (47\%) did not show demyelinating features. Eleven(37\%)

${ }^{1} \mathrm{C}$. Mondino National Neurological Institute, Department of Neurological Sciences, 27100, Pavia, Italy

Full list of author information is available at the end of the article had a negative tissue biopsy and 4/5(80\%) had raised proteins in cerebrospinal fluid (CSF). We next compared EDx of 53 ATTR with EDx from 35 matched CIDP patients. Conduction slowing and prolongation of distal motor latencies were less prominent in ATTR vs CIDP while conduction blocs were almost invariably absent in ATTR. Conversely, in ATTR motor nerves were more often not excitable both at upper and lower limbs.

\section{Conclusions}

ATTR was misdiagnosed in $1 / 3$ of cases, particularly in patients with late onset and without family history. CIDP was the most common alternative diagnosis, which was supported by EFNS EDx criteria for demyelinating neuropathy in half of them. However conduction slowing is less prominent in ATTR while severe axonal loss is the major EDx feature. DNA testing for TTR should be performed in patients with progressive axonal or mixed axonaldemyelinating peripheral neuropathy, who do not respond to immunotherapies, regardless the lack of family history and a late onset. Raised proteins in CSF and a negative biopsy do not rule out the diagnosis of ATTR.

\section{Authors' details}

${ }^{1} \mathrm{C}$. Mondino National Neurological Institute, Department of Neurological Sciences, 27100, Pavia, Italy. ${ }^{2}$ Amyloidosis Research and Treatment Center, Department of Molecular Medicine, Fondazione Istituto Di Ricovero e Cura a Carattere Scientifico Policlinico San Matteo and University of Pavia, 27100, Pavia, Italy.

Published: 2 November 2015

doi:10.1186/1750-1172-10-S1-013

Cite this article as: Cortese et al:: Misdiagnoses of transthyretin amyloidosis: a clinical and electrodiagnostic study. Orphanet Journal of Rare Diseases 2015 10(Suppl 1):013. 